\title{
EVALUACIÓN DE CULTIVARES DE ARROZ (Oryza sativa L.) BAJO CONDICIONES DE SECANO Y RIEGO. PANAMÁ. 2002-20031
}

\author{
Ismael Camargo Buitrago ${ }^{2}$, Luisa Martínez Rodríguez ${ }^{3}$, Eric Batista Montero ${ }^{3}$, Pedro Him Him ${ }^{3}$, \\ Eric Quirós Rodríguez $z^{3}$, Benjamín Name Tuñón ${ }^{3}$
}

\begin{abstract}
RESUMEN
Evaluación de cultivares de arroz (Oryza sativa $\mathbf{L}$.) bajo condiciones de secano y riego. Panamá. 2002-2003. Se evaluó la estabilidad (AMMI y el Biplot $\mathrm{GGE}_{\mathrm{SREG}}$ ) y otras características de 20 genotipos de arroz, en secano y riego. Para el sistema de secano, hubo diferencias en rendimiento, sobresaliendo FLAR 54-00, IDIAP 38 y Lajas 02-00. En calidad de grano, el rendimiento total varió entre 61,8 a $68,0 \%$, los granos enteros entre 43,6 y $56,5 \%$, y el centro blanco varió entre 1,5 y 3,4. Para la digestión alcalina, diez genotipos mostraron grados de dispersión entre seis y siete con baja temperatura de gelatinización, ocho con grado de cuatro a cinco considerados intermedios. El FLAR 54-00 respondió mejor a los estímulos ambientales. Prosequisa, CHI 32-10 y CHI 45-23, presentaron el comportamiento más pobre en la mayoría de ambientes. El modelo Biplot GGE $\mathrm{SREG}_{\text {, }}$ identificó como genotipos estables, al FLAR 54-00, FLAR 198-00 y Fedearroz 50. Para el sistema de riego, hubo diferencias en rendimiento, destacándose IDIAP 38, P-3621 y Fedearroz 50. La calidad de granos, mostró rendimiento total entre 65,8 a $70,9 \%$, granos enteros entre 51,5 y $62,0 \%$, la incidencia de centro blanco varió entre 1,2 y 2,8 . En digestión alcalina, nueve genotipos presentaron grados de seis y siete, y diez genotipos con grados de cuatro a cinco. IDIAP 38, P3621 y FLAR 54-00, respondieron mejor a los estímulos ambientales. Prosequisa, FLAR 195-00, CHI 49-16 y CHI 13256, presentaron el comportamiento más pobre. Los genotipos más estables bajo riego, fueron Fedearroz 50, IDIAP 38 y P-3621.
\end{abstract}

Palabras clave: Arroz, variedades, rendimiento, estabilidad, evaluación de cultivares, Biplot GGE.

\begin{abstract}
Evaluation of cultivated of rice (Oryza sativa L.) under conditions of dry land and irrigation. Panamá. 2002-2003. The stability and other characteristics of 20 genotypes of rice, grown under dry-land and irrigation conditions, were evaluated. Statistical analyses were conducted (AMMI and Biplot GGESREG) to estimate stability. For the dryland system, there were differences in yield, excelling FLAR 54-00, 38 IDIAP and Lajas 02-00. Total yield fluctuated between 61.8 and $68.0 \%$, entire grains between 43.6 and $56.5 \%$ and the white center between 1.5 and $3.4 \%$. The alkaline digestion showed ten genotypes with degrees of dispersion between six and seven with low temperature of gelatinización, eight with degrees from 4 to 5 considered intermediate. FLAR 54-00 responded better to the environmental. Prosequisa, CHI 32-10 and CHI 45-23 showed the poorest behavior. The model Biplot GGESREG identified FLAR 54-00, FLAR 198-00 and Fedearroz 50 as stable genotypes. For the irrigation system, statistical DMS $(\mathrm{P}<0.05)$ indicated differences in yield, standing out IDIAP 38, P-3621 and Fedearroz 50. The quality of grains showed total yield between $65.8-70.9 \%$, entire grains between 51.5 and $62.0 \%$, and incidence of white center between 1.2 and $2.8 \%$. In the alkaline digestion, nine genotypes presented degrees of six and seven and ten with degrees from 4 to 5. IDIAP 38, P-3621 and FLAR 54-00, responded better to the environmental stimuli. Prosequisa, FLAR 195-00, CHI 49-16 and CHI 13256 presented the poorest behavior. The stable genotypes under irrigation were Fedearroz 50, 38 IDIAP and P-3621.
\end{abstract}

Key words: Rice, genotype, yield, stability, evaluation of cultivars, Biplot GGE.

\footnotetext{
1 Recibido: 19 de octubre, 2004. Aceptado: 30 de agosto, 2005. Presentado en la L Reunión Anual del PCCMCA. El Salvador. 2004.

2 Coordinador del Proyecto de arroz del Instituto de Investigación Agropecuaria de Panamá (IDIAP), Panamá. E-mail: icamargo@cwpanama.net

3 Equipo técnico de investigadores de arroz del Instituto de Investigación Agropecuaria de Panamá (IDIAP).
} 


\section{INTRODUCCIÓN}

El arroz es el grano más importante en la dieta del panameño, esto se refleja en el alto consumo. De acuerdo al Ministerio de Desarrollo Agropecuario (MIDA 2003), se consumen mensualmente unas $21.411,5$ toneladas de arroz en cáscara. En el año 2002, para suplir esta demanda en el país, se sembraron 74.752 hectáreas. En esta actividad participaron unos 1.381 productores, que cultivaron 60.824 hectáreas en secano $(81,4 \%)$, mientras 122 productores plantaron 13,928 hectáreas con riego $(18,6 \%)$. El rendimiento promedio nacional en el 2003 fue de 5.517 toneladas por hectárea.

Los cultivares experimentales y comerciales de arroz, son evaluados en numerosas localidades y años, donde diferentes características agronómicas e industriales son utilizadas para la selección de los cultivares superiores. Para recomendarlos a los productores y también para identificar los sitios para la siembra en las regiones de interés. Cuando efectuamos estos trabajos de investigación nos enfrentamos al fenómeno de la interacción genotipo x ambiente ( $\mathrm{G} \times \mathrm{A}$ ), el cual afecta el comportamiento de los genotipos cuando son evaluados a través de ambientes contrastantes (localidades, épocas, años, etc.), dificultando la identificación y selección de los genotipos superiores. Al estudio de este fenómeno es necesario integrar los conceptos de adaptabilidad y estabilidad, para definir el comportamiento de genotipos. La adaptabilidad se refiere a la capacidad de los genotipos de aprovechar ventajosamente los estímulos del ambiente; en cuanto que la estabilidad, se refiere a la capacidad de los genotipos de mostrar un comportamiento altamente previsible en función del estímulo ambiental (Lin et al. 1986; Lin y Binns 1994).

El desarrollo del modelo AMMI (Efectos principales aditivos e interacción multiplicativa), ha contribuido a un mejor entendimiento de este fenómeno (Zobel et al. 1988; Gauch y Zobel 1988; Crossa 1990; Crossa et al. 1990; Crossa et al. 1991).

Yan et al. 2000, propuso el modelo Biplot GGE. Este modelo se refiere al efecto principal de genotipo (G) más la interacción genotipo por ambiente (GE), siendo estas las dos fuentes de variación más relevantes en la evaluación de cultivares proveniente de ensayos en múltiples localidades y años. En términos generales la grafica Biplots GGE permite visualizar las similitudes y diferencias entre localidades y la respuesta diferencial de los genotipos; las similitudes y diferencias entre genotipos en su respuesta a las diferentes localidades; y la naturaleza (positiva vs. negativa), así como la magnitud de la interacción entre cualquier genotipo y cualquier localidad (Yan et al. 2000; Yan et al. 2001; Yan y Rajcan 2002).

\section{Objetivos}

Seleccionar las líneas avanzadas y variedades introducidas de arroz que igualen o superen a las mejores variedades comerciales por medio de la evaluación en diferentes áreas arroceras en los sistemas de secano y de riego, para satisfacer la demanda de los productores, procesadores y consumidores de este grano.

\section{MATERIALES Y MÉTODOS}

\section{Ubicación}

El experimento se realizó en diez localidades en el sistema de secano y seis bajo riego en las principales regiones productoras de arroz de Panamá, la mayoría en parcelas facilitadas por productores colaboradores durante el ciclo de siembra comprendido entre los meses de junio a diciembre, correspondiente al año 2002 (Cuadro 1).

\section{Material genético}

Los grupos de cultivares evaluados provienen de diferentes empresas del sector público y privado. El Oryzica 1 , fue el testigo para evaluar la calidad de grano (Cuadro 2).

\section{Manejo agronómico}

El manejo se efectuó de acuerdo a la tecnología generada por el Instituto de Investigación Agropecuaria de Panamá (IDIAP) en las diferentes regiones productoras. La rata de siembra (cantidad de semilla utilizada por metro lineal, equivalente a 150 kilogramos de semilla por hectárea) fue de tres gramos por metro lineal. Las enfermedades no se controlaron para permitir la diferenciación genética de los genotipos e igualmente las plagas fueron controladas en casos de extrema necesidad, cuando en la floración se tienen poblaciones de insectos (Oebalus sp.) sobre los umbrales económicos. El control se hizo para que estos insectos no causaran lesiones en el grano, de manera que no favoreció la incidencia del manchado del grano ocasionado por hongos y bacterias.

El sistema de secano, fue dependiente totalmente de la precipitación. En el sistema con riego se mantuvo, como mínimo, un riego por inundación cada semana. En algunas localidades fue posible mantener una lámina de agua de 5 a $10 \mathrm{~cm}$ de profundidad a partir de los 15 días después de siembra. 
Cuadro 1. Ubicación de las localidades donde se efectuaron las evaluaciones de los 20 cultivares de arroz en los sistemas de secano y riego. IDIAP. Panamá. 2002-2003.

\begin{tabular}{|c|c|c|c|c|c|c|c|}
\hline \multicolumn{4}{|c|}{ Sistema de secano } & \multicolumn{4}{|c|}{ Sistema de riego } \\
\hline $\mathbf{N}^{\circ}$ & Localidades & Distrito & Provincia & $\mathbf{N}^{\circ}$ & Localidades & Distrito & Provincia \\
\hline 1 & Campo Expe. & Alanje & Chiriquí & 1 & Canta Gallo & Alanje & Chiriquí \\
\hline 2 & La Esperanza & Barú & Chiriquí Occidente & 2 & Jacú & Barú & Chiriquí Occidente \\
\hline 3 & Calabacito & San Francisco & Veraguas & 3 & Remedios & Remedios & Chiriquí Oriente \\
\hline 4 & Divisa & Santa María & Herrera & 4 & San Lorenzo & San Juan & Chiriquí Oriente \\
\hline 5 & INA & Cañazas & Veraguas & 5 & Sup. Campesina & Chepo & Panamá Este \\
\hline 6 & Remedios & Remedios & Chiriquí Oriente & 6 & El Coco & Penonomé & Coclé \\
\hline 7 & Sup. Campesina & Chepo & Panamá Este & & & & \\
\hline 8 & Trinchera & Soná & Veraguas & & & & \\
\hline 9 & El Coco & Penonomé & Coclé & & & & \\
\hline 10 & El Cacao & Tonosí & Los Santos & & & & \\
\hline
\end{tabular}

\section{Diseño experimental y análisis estadístico}

Se utilizó un diseño de bloques completos al azar, con tres repeticiones. La unidad experimental constó de una parcela de 10 hileras de $5 \mathrm{~m}$ de longitud, separadas a 0,20 m. La parcela efectiva estuvo constituida por las seis hileras centrales a las que se eliminó $0,50 \mathrm{~m}$ de borde en cada extremo $\left(4,8 \mathrm{~m}^{2}\right)$.

Se realizó un análisis de varianza combinado, considerando un modelo mixto (ambiente aleatorio y genotipo fijo). Para la separación de las medias se utilizó la diferencia mínima significativa $(\mathrm{DMS}=\mathrm{P} \leq 0,05)$.

\section{Análisis de estabilidad}

Para el análisis de estabilidad se utilizó el modelo efectos principales aditivos e interacción multiplicativa
(AMMI), que integra el análisis de varianza y el de componentes principales (Zobel et al. 1988).

\section{Interpretación de la interacción genotipo $\mathrm{x}$ ambien- te mediante la gráfica Biplot GGE $_{\text {SREG }}$}

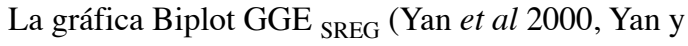
Hunt 2002) permite: 1. determinar el cultivar con mejor comportamiento en un ambiente específico; 2 . La identificación del ambiente más apropiado para un cultivar específico; 3. La comparación de cualquier par de cultivares en un ambiente; 4. El mejor cultivar para cada ambiente y la diferenciación de mega ambientes. Yan et al. 2001, mostró que este gráfico permitía identificar además al genotipo ideal como aquel con alta puntuación en el primer eje del componente principal (PCA1), asociada a altos rendimientos y las puntuaciones cercanas a cero en el segundo eje del componente principal

Cuadro 2. Listado de los 20 cultivares de arroz y su origen, evaluados bajo las condiciones de secano y riego. IDIAP 2002-03.

\begin{tabular}{|c|c|c|c|c|c|c|c|c|c|}
\hline $\mathbf{N}^{\circ}$ & & Tratamiento & $\begin{array}{l}\text { Institución } \\
\text { obstentora }\end{array}$ & $\begin{array}{l}\text { País de } \\
\text { origen }\end{array}$ & $\mathbf{N}^{\circ}$ & & Tratamiento & $\begin{array}{l}\text { Institución } \\
\text { obstentora }\end{array}$ & $\begin{array}{c}\text { País de } \\
\text { origen }\end{array}$ \\
\hline 1 & P 21 & P-3621 & FCA-IDIAP-CIAT & Panamá & 11 & C 03 & CHI 3-30 & IDIAP & Panamá \\
\hline 2 & I 22 & IDIAP 22 & IDIAP & Panamá & 12 & C 32 & CHI 32-10 & IDIAP & Panamá \\
\hline 3 & F 50 & FEDEARROZ 50 & FEDEARROZ & Panamá & 13 & I 38 & IDIAP 38 & IDIAP & Panamá \\
\hline 4 & O 1 & Oryzica & ICA-CIAT & Colombia & 14 & P 48 & P-1048 & FCA-IDIAP-CIAT & Panamá \\
\hline 5 & PR & PROSEQUISA & & Rep. Dominicana & 15 & $\mathrm{~L} 02$ & LAJAS 02-00 & IDIAP & Panamá \\
\hline 6 & F 54 & FLAR 54-00 & IDIAP/FLAR/FEDAGPA & Panamá & 16 & C 49 & CHI 49-6 & IDIAP & Panamá \\
\hline 7 & F 99 & FLAR 199-00 & IDIAP/FLAR/FEDAGPA & Panamá & 17 & C 75 & CHI 75-25 & IDIAP & Panamá \\
\hline 8 & F 95 & FLAR 195-00 & IDIAP/FLAR/FEDAGPA & Panamá & 18 & V 30 & VIORH 30 & IDIAP & Panamá \\
\hline 9 & F 98 & FLAR 198-00 & IDIAP/FLAR/FEDAGPA & Panamá & 19 & C 13 & CHI 13-256 & IDIAP & Panamá \\
\hline 10 & C 45 & CHI 45-23 & IDIAP & Panamá & 20 & C 16 & CHI 16-12 & IDIAP & Panamá \\
\hline
\end{tabular}


(PCA2), está relacionado con buena estabilidad del cultivar a través de los ambientes contrastantes. El mismo criterio se aplica para determinar el ambiente ideal.

Además en el Biplot GGE, los genotipos que se encuentran ubicados hacia el centro de la figura son menos responsivos que aquellos localizados en las esquinas o vértices del polígono los cuales se consideran más responsivos (positiva o negativamente). También pueden ser identificados los mejores y peores sitios, esta información es utilizada para identificar los mega-ambientes en las regiones de interés. Los cultivares ubicados en los sectores donde no hay localidades son consideradas de pobre comportamiento en rendimiento en la mayoría de las localidades testadas (Yan et al. 2000; Yan et al. 2001; Yan y Rajcan 2002; Kaya et al. 2002).

\section{Características evaluadas}

Las variables evaluadas se calificaron de acuerdo al Sistema de Evaluación Estándar para Arroz. IIRICIAT. Basados en la escala de uno a nueve; donde: 1 ideal; 3 aceptable; 7 a 9 indeseable. La evaluación de la calidad del grano se efectuó de acuerdo a la metodología propuesta por el CIAT (Arregocés y Zamorano 1979). A continuación se indican las variables evaluadas:

\begin{tabular}{|c|c|c|}
\hline Vigor (VG) & Hoja blanca (HB) & Bacteriosis (BB) \\
\hline Altura de planta (HT) & Escaldado (LSC) & $\begin{array}{l}\text { Rendimiento de gra- } \\
\text { no t/ha al } 14 \% \text { de } \\
\text { humedad (REND) }\end{array}$ \\
\hline $\begin{array}{l}\text { Resistencia al acame } \\
\text { (LDG) }\end{array}$ & $\begin{array}{l}\text { Manchado del grano } \\
\text { (GID) }\end{array}$ & $\begin{array}{l}\text { Rendimiento total } \\
\text { (RT) }\end{array}$ \\
\hline Días a floración (FL) & Rhyzoctonia (SHB) & Granos enteros (GE) \\
\hline $\begin{array}{l}\text { Días a maduración } \\
\text { (Mat) }\end{array}$ & $\begin{array}{l}\text { Mancha ojival por } \\
\text { dreshlera (MO) }\end{array}$ & $\begin{array}{l}\text { Granos quebrados } \\
\text { (GQ) }\end{array}$ \\
\hline $\begin{array}{l}\text { Piricularia al follaje } \\
\text { (BI) }\end{array}$ & $\begin{array}{l}\text { Cercosporiosis } \\
\text { (NBLS) }\end{array}$ & $\begin{array}{l}\text { Centro blanco } \\
\text { (CB) }\end{array}$ \\
\hline $\begin{array}{l}\text { Piricularia al cuello } \\
\text { de la panoja (NBI) }\end{array}$ & $\begin{array}{l}\text { Sarocladium } \\
\text { (SHR) }\end{array}$ & $\begin{array}{l}\text { Digestión alcalina } \\
\text { (DA) }\end{array}$ \\
\hline $\begin{array}{l}\text { Helminthosporium } \\
\text { (BS) }\end{array}$ & Falso carbón (FSm) & \\
\hline
\end{tabular}

\section{RESULTADOS Y DISCUSIÓN}

\section{Resultados en el sistema de secano}

\section{Condiciones ambientales}

El 2002 se caracterizó por un comportamiento errático de las lluvias específicamente en el litoral pacífico del país, influenciado por el fenómeno del "NIÑO" (ENOS). Esta situación afectó las siembras comerciales especialmente las tardías, puesto que las lluvias se suspendieron a mediados de noviembre en todo el país, afectando significativamente el rendimiento de grano, como fue el caso de las siembras atrasadas de Coclé, Tonosí y las realizadas en Chiriquí durante el segundo semestre (Figura 1).

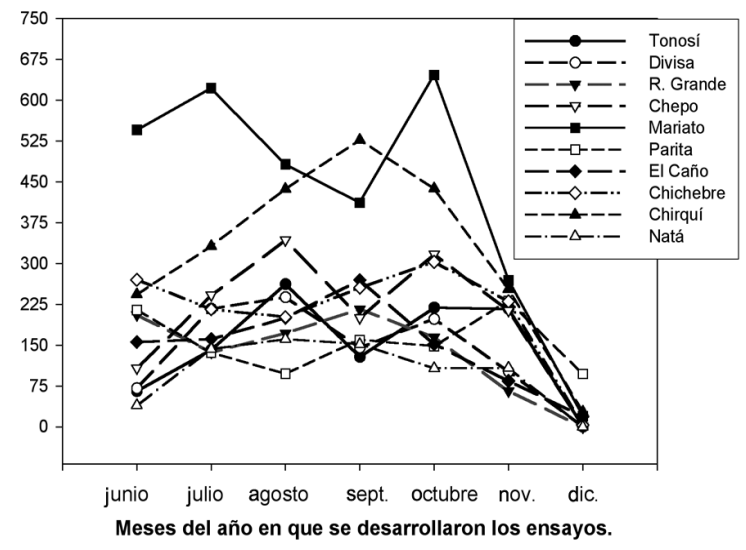

Figura 1. Distribución mensual de las lluvias en diez localidades del litoral pacífico de Panamá. 2002.

Análisis de varianza e interpretación de la interacción genotipo - ambiente

El análisis de varianza AMMI (Cuadro 3), muestra que el $81,2 \%$ de la suma de cuadrado total fue atribuible a los efectos ambientales, 3,3\% a los efectos de genotipos y $15,4 \%$ a la interacción G x A. La mayor suma de cuadrado para ambientes indica, que los ambientes fueron diferentes y contrastantes con diferencias en magnitud entre las medias ambientales, ocasionando variaciones en el rendimiento de grano. La magnitud de la suma de cuadrados de la interacción $\mathrm{G}$ x A, fue 4,7 veces mayor que la de genotipo, indicando que hubieron diferencias substanciales en la respuesta de los genotipos a través de los ambientes, con interacciones cruzadas.

En el Cuadro 3, se observa que ambos ejes están explicando el $67 \%$ de la interacción genotipo por ambiente, el PCA-1 explica el 44\% de la interacción y el PCA-2 captura el $23 \%$ restante.

\section{Efectos de genotipos}

De acuerdo a la gráfica Biplots GGE (Figura 2) los genotipos que más respondieron a los estímulos ambientales positiva o negativamente, dependiendo del signo y la magnitud de la puntuación, fueron el IDIAP 38, CHI 45-23, Prosequisa, FLAR 199-00 y FLAR 19800 y FLAR 54-00. 
Cuadro 3. Análisis AMMI, para rendimiento de grano de 20 cultivares de arroz evaluados en diez ambientes bajo condiciones de secano. IDIAP. Panamá. 2002.

\begin{tabular}{|c|c|c|c|c|}
\hline $\begin{array}{l}\text { Fuente de } \\
\text { variación }\end{array}$ & $\begin{array}{c}\text { Grados de } \\
\text { libertad }\end{array}$ & $\begin{array}{l}\text { Suma de } \\
\text { cuadrado }\end{array}$ & $\begin{array}{c}\text { Cuadrado } \% \\
\text { medio }\end{array}$ & $\begin{array}{l}\% \text { explicado } \\
\text { por fuente } \\
\text { var. }\end{array}$ \\
\hline Modelo & 201 & 1861,697250 & $9,262175^{* *}$ & \\
\hline Ambientes (A) & 9 & 1512,409276 & $168,045475^{* *}$ & 81,2 \\
\hline Genotipos (G) & 19 & 60,458486 & $3,182026^{* *}$ & 3,3 \\
\hline $\mathrm{G} \times \mathrm{A}$ & 171 & 286,029861 & $1,672689 * *$ & 15,4 \\
\hline PCA1 & 27 & 129,302 & 4,78896 & 44 \\
\hline PCA2 & 25 & 57,626 & 2,30503 & 23 \\
\hline Error & 398 & 282,041276 & 0,708646 & \\
\hline Total & 599 & 2143,738526 & & \\
\hline
\end{tabular}

La línea avanzada FLAR 54-00, fue considerada como el genotipo que mejor respondió a los estímulos ambientales concepto de adaptabilidad de Lin et al. (1986), Lin y Binns (1994), específicamente tuvo buen desempeño en las localidades de Alanje, Calabacito, Divisa, Remedios, Tanara, Trinchera y Tonosí.

Los cultivares Prosequisa, CHI 32-10 y CHI 4523, fueron los que presentaron el comportamiento mas pobre en la mayoría de los ambientes, especialmente la variedad Prosequisa de ciclo tardío y las condiciones climáticas del ciclo 2002 fueron muy adversas para este material.
Los genotipos con mejor estabilidad fueron FLAR 54-00, FLAR 198-00 y Fedearroz 50, por presentar rendimientos sobre la media del ensayo, puntuaciones altas en el PCA1 y puntuaciones cercanas a cero en el PCA2. Estas características, de acuerdo al modelo, identifican los cultivares mas estables a través de localidades (Figura 2).

\section{Efectos de los ambientes}

Los ambientes fueron agrupados en tres sectores. El primero conformado únicamente por Penonomé, en el segundo se ubican las localidades de Barú, Remedios, Trinchera, Tanara y Divisa, y en el tercer sector se ubicaron las localidades de Alanje, INA, Calabacito, y Tonosí, donde el genotipo superior fue el FLAR 54-00.

La localidades Penonomé, Barú y Calabacito fueron las que presentaron la mayor capacidad discriminatoria de los cultivares, indicado por la longitud de los vectores de estas localidades.

\section{Análisis de medias}

En el Cuadro 4, se presentan las medias de algunas de las variables en estudio. Es importante destacar que las características cuantitativas están expresadas en términos de la media y la desviación estándar, mientras que las características basadas en escalas de evaluación, está expresada por el valor medio y entre el paréntesis la

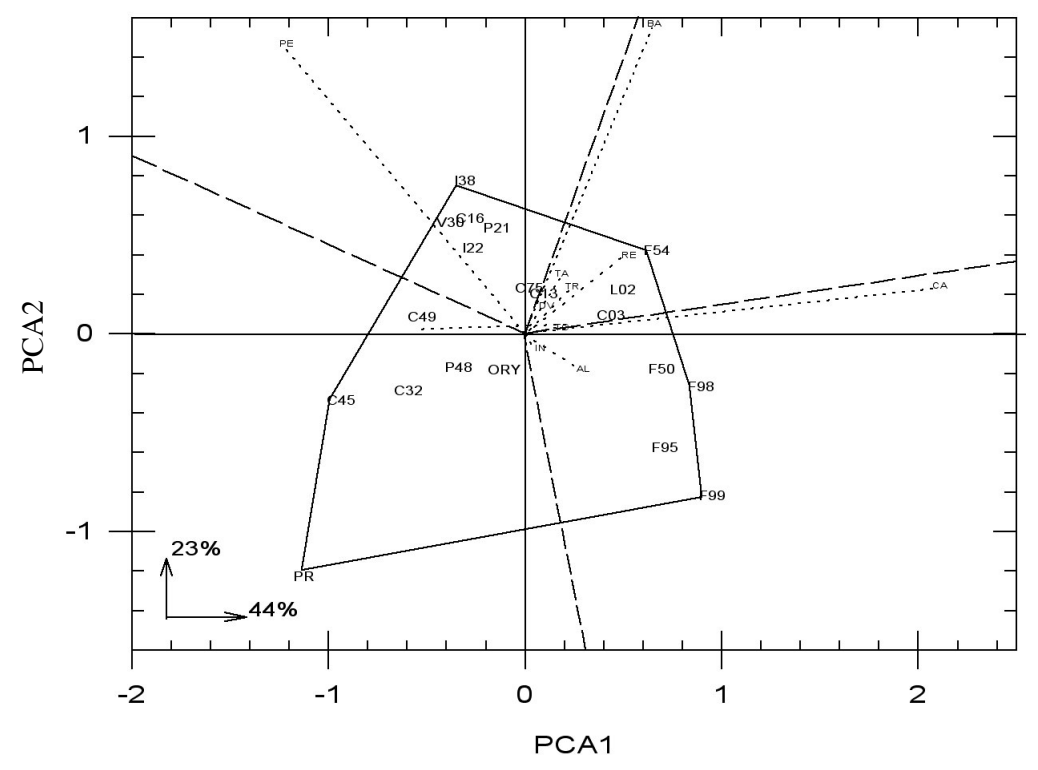

Figura 2. Puntuaciones del primer y segundo eje del componente principal de 20 cultivares de arroces intermedios en 10 ambientes de Secano. Panamá, 2002. (GGEBiplot). 
Cuadro 4. Rendimiento promedio t/ha (14\%) de humedad y otras características agronómicas e industriales de 20 cultivares de arroz bajo el sistema de secano, evaluadas en 10 localidades. IDIAP, Panamá. 2002-2003.

\begin{tabular}{|c|c|c|c|c|c|c|c|c|c|c|}
\hline Cultivar & $\begin{array}{l}\text { HT } \\
(\mathbf{c m})\end{array}$ & $\begin{array}{c}\text { FL } \\
\text { (dds) }\end{array}$ & $\begin{array}{c}\text { BL } \\
(1-9)\end{array}$ & $\begin{array}{l}\text { NBL } \\
(1-9)\end{array}$ & $\begin{array}{l}\text { LSC } \\
(1-9)\end{array}$ & $\begin{array}{c}\text { REND } \\
\text { (t/ha) }\end{array}$ & $\begin{array}{l}\text { RT } \\
(\%)\end{array}$ & $\begin{array}{l}\text { GE } \\
(\%)\end{array}$ & $\begin{array}{c}\text { CB } \\
(1-5)\end{array}$ & $\begin{array}{c}\text { DA } \\
(1-7)\end{array}$ \\
\hline FLAR 54-00 & $87 \pm 10$ & $88 \pm 10$ & $2(6)$ & 2(3) & $3(7)$ & $3.289 \pm 2,01$ & $67,5 \pm 5,4$ & $51,2 \pm 15,1$ & $2,7 \pm 1,4$ & $6,8 \pm 0,3$ \\
\hline IDIAP 38 & $83 \pm 9$ & $92 \pm 7$ & $3(8)$ & $3(9)$ & $3(7)$ & $2.971 \pm 1,92$ & $65,4 \pm 5,4$ & $50,0 \pm 12,0$ & $2,3 \pm 1,4$ & $3,6 \pm 0,3$ \\
\hline LAJAS 02-00 & $90 \pm 11$ & $94 \pm 9$ & $2(5)$ & 2(3) & $3(7)$ & $2.952 \pm 1,99$ & $63,9 \pm 6,5$ & $51,7 \pm 12,1$ & $2,6 \pm 1,5$ & $6,7 \pm 0,3$ \\
\hline CHI $75-25$ & $84 \pm 12$ & $90 \pm 10$ & $2(5)$ & $3(9)$ & $3(7)$ & $2.840 \pm 2,07$ & $66,6 \pm 4,3$ & $49,9 \pm 13,2$ & $2,6 \pm 1,1$ & $6,2 \pm 0,9$ \\
\hline CHI 16-12 & $90 \pm 12$ & $91 \pm 12$ & $2(5)$ & $3(7)$ & $3(7)$ & $2.778 \pm 2,08$ & $65,6 \pm 4,0$ & $50,5 \pm 9,3$ & $3,3 \pm 0,8$ & $3,9 \pm 0,9$ \\
\hline FLAR 198-00 & $86 \pm 13$ & $88 \pm 12$ & $2(5)$ & 2(4) & $3(9)$ & $2.732 \pm 1,99$ & $67,7 \pm 4,0$ & $51,1 \pm 15,6$ & $2,3 \pm 0,9$ & $5,8 \pm 1,3$ \\
\hline FLAR 195-00 & $87 \pm 13$ & $86 \pm 11$ & $2(7)$ & $2(5)$ & $3(9)$ & $2.714 \pm 1,93$ & $68,0 \pm 4,6$ & $53,2 \pm 11,5$ & $1,5 \pm 0,3$ & $6,9 \pm 0,1$ \\
\hline FEDEARROZ 50 & $90 \pm 12$ & $94 \pm 10$ & $2(5)$ & $2(5)$ & $3(7)$ & $2.706 \pm 2,00$ & $66,5 \pm 5,8$ & $54,3 \pm 10,7$ & $2,2 \pm 1,3$ & $6,7 \pm 0,3$ \\
\hline CHI $13-256$ & $88 \pm 11$ & $90 \pm 10$ & $2(6)$ & $3(5)$ & $3(9)$ & $2.683 \pm 1,80$ & $66,7 \pm 4,7$ & $49,2 \pm 11,6$ & $2,9 \pm 1,1$ & $5,7 \pm 1,4$ \\
\hline CHI 3-30 & $86 \pm 11$ & $98 \pm 8$ & $2(4)$ & $2(5)$ & $3(7)$ & $2.620 \pm 1,76$ & $63,4 \pm 5,4$ & $47,7 \pm 11,3$ & $2,5 \pm 1,4$ & $4,0 \pm 0,1$ \\
\hline ORYZICA 1 & $83 \pm 11$ & $87 \pm 11$ & $3(9)$ & $3(9)$ & $4(9)$ & $2.620 \pm 1,75$ & $64,713,2$ & $50,5 \pm 17,2$ & $1,7 \pm 0,6$ & $6,0 \pm 1,0$ \\
\hline VIORH 30 & $92 \pm 13$ & $96 \pm 8$ & $2(7)$ & $2(7)$ & $3(7)$ & $2.617 \pm 1,73$ & $64,9 \pm 5,3$ & $50,0 \pm 9,5$ & $2,7 \pm 1,2$ & $4,4 \pm 0,4$ \\
\hline P-3621 & $87 \pm 11$ & $99 \pm 6$ & $3(8)$ & $2(7)$ & $3(7)$ & $2.598 \pm 1,88$ & $63,3 \pm 6,8$ & $46,1 \pm 15,5$ & $2,5 \pm 1,2$ & $6,2 \pm 1,4$ \\
\hline IDIAP 22 & $89 \pm 11$ & $99 \pm 6$ & $2(7)$ & 2(9) & $3(7)$ & $2.547 \pm 1,77$ & $64,6 \pm 6,6$ & $50,1 \pm 13,7$ & $2,7 \pm 1,3$ & $6,4 \pm 1,1$ \\
\hline FLAR 199-00 & $87 \pm 15$ & $90 \pm 11$ & $2(5)$ & $2(5)$ & $3(9)$ & $2.545 \pm 2,07$ & $68,0 \pm 3,9$ & $56,5 \pm 7,9$ & $1,7 \pm 0,4$ & $6,9 \pm 0,1$ \\
\hline CHI 49-6 & $93 \pm 11$ & $97 \pm 9$ & $3(7)$ & $2(5)$ & $3(7)$ & $2.490 \pm 1,78$ & $66,0 \pm 6,1$ & $55,0 \pm 11,0$ & $1,9 \pm 0,6$ & $5,2 \pm 0,9$ \\
\hline P-1048 & $87 \pm 10$ & $97 \pm 8$ & $3(8)$ & $3(9)$ & $3(7)$ & $2.465 \pm 1,86$ & $65,7 \pm 6,1$ & $49,3 \pm 13,2$ & $2,2 \pm 0,9$ & $6,9 \pm 0,1$ \\
\hline CHI 45-23 & $82 \pm 11$ & $95 \pm 8$ & $3(6)$ & 2(9) & $4(7)$ & $2.302 \pm 2,05$ & $63,2 \pm 5,9$ & $47,8 \pm 10,5$ & $2,6 \pm 1,4$ & $4,1 \pm 0,1$ \\
\hline CHI $32-10$ & $83 \pm 12$ & $97 \pm 8$ & $4(7)$ & $3(9)$ & $3(7)$ & $2.290 \pm 1,61$ & $61,8 \pm 6,4$ & $43,6 \pm 13,1$ & $3,4 \pm 1,2$ & $4,0 \pm 0,4$ \\
\hline PROSEQUISA & $101 \pm 15$ & $102 \pm 8$ & $3(8)$ & 2(9) & $3(7)$ & $1.637 \pm 1,75$ & $64,5 \pm 5,6$ & $55,4 \pm 10,0$ & $2,1 \pm 1,4$ & $5,4 \pm 1,6$ \\
\hline Promedio & 88 & 93 & 2 & 2 & 3 & 2.620 & 65,4 & $\mathbf{5 0 , 7}$ & 2,4 & 5,6 \\
\hline DMS & 3 & 2 & 0,6 & 0,5 & 0,5 & 0,419 & & & $2,7 \pm 1,4$ & $6,8 \pm 0,3$ \\
\hline
\end{tabular}

máxima calificación obtenida en una localidad específica. Los resultados muestran que la floración bajo las condiciones de secano fluctuó de $87 \pm 11$ días en Oryzica 1 hasta los $102 \pm 8$ días después de siembra en la Prosequisa, con medias general del ensayo de 93 días.

Se observó baja incidencia de enfermedades como la Piricularia al follaje (Bl) y al cuello de la panícula (NBl) y ataques moderado de escaldado de la hoja (LSc). La baja incidencia promedio de enfermedades en las parcelas experimentales no indica tolerancia absoluta de los genotipos, los paréntesis indican la evaluación máxima alcanzada por estos genotipos, sugiriendo que se debe tener cuidado con la incidencia de estas enfermedades en estos cultivares a corto y mediano plazo.

Hubo diferencias en rendimiento de grano, sobresaliendo FLAR 54-00, IDIAP 38 y LAJAS 02-00, las tres presentaron porcentaje de granos enteros (GE) igual o sobre la media del experimento, que fue de 50 $\%$. Es importante destacar que el bajo rendimiento promedio obtenido $(2.620 \mathrm{t} / \mathrm{ha})$, se debe en parte a las localidades de Divisa y el INA, afectadas por sequía con medias de 0,195 y 0,46 t/ha respectivamente, ambas están incluidas en el análisis combinado.
Las condiciones de secano no fueron favorables para la calidad del grano, registrándose porcentajes de rendimiento total (RT) entre 61,8 a $68,0 \%$, mientras los valores de granos enteros (GE) oscilaron entre 43,6 y $56.5 \%$. Por otro lado, los rangos de centro blanco (CB) en los granos fluctuó entre 1,5 y 3,4 en la escala de uno a cinco, estos valores de $\mathrm{CB}$, están relativamente altos. Destacándose además, que los materiales más rendidores presentaron valores de CB superiores a 2,3, tal vez influenciado por las características genéticas y por las condiciones adversas después de la antesis o por deficiencias en el manejo post cosecha.

En cuanto a la digestión alcalina (DA), diez genotipos presentaron grados de dispersión entre seis y siete con baja temperatura de gelatinización, ocho con grado de cuatro a cinco considerados intermedios, y dos con grado inferior a cuatro con alta temperatura de gelatinización. La temperatura de gelatinización baja e intermedia indican que el arroz tuvo buena absorción de agua y quedará suelto y seco después de cocido. Por el contrario, temperatura de gelatinización alta normalmente indica que quedará pegajoso después de cocido (Arregocés y Zamorano 1979). 


\section{Resultados en el sistema de riego}

\section{Condiciones ambientales}

El sistema de riego consistió básicamente en mantener las parcelas experimentales a capacidad de campo (mínimo un riego semanal), este fue el manejo practicado en las localidades de Remedios, Tanara y Alanje; mientras que en Jacú, San Lorenzo y Penonomé se mantuvo una lámina de agua a partir de los 15 días después de siembra.

\section{Análisis de varianza e interpretación de la interac- ción genotipo $x$ ambiente}

El análisis de varianza AMMI (Cuadro 5), para rendimiento de grano muestra que el $81 \%$ de la suma de cuadrado total fue atribuible a los efectos ambientales, $6 \%$ a los efectos genotipos y $12 \%$ a la interacción $\mathrm{G} \times \mathrm{A}$. La mayor suma de cuadrado para ambiente indica que los ambientes o sistemas de manejo del agua fueron diferentes en las localidades, con diferencias entre las medias ambientales y variaciones en el rendimiento de grano como respuesta de los genotipos. La magnitud de la suma de cuadrados de la interacción $\mathrm{G}$ $\mathrm{x} A$, fue dos veces mayor que la de genotipo, indicando que hubieron respuestas diferenciales de los genotipos a través de los ambientes. Los cultivares con un comportamiento excelente en una localidad no repitieron ese comportamiento en los otros ambientes.
Además los dos ejes del componente principal están explicando el $63 \%$ de la interacción genotipo por ambiente, el PCA-1 explica el 43\% de la interacción y el PCA-2 que captura el $20 \%$ (Cuadro 5).

\section{Efectos de los genotipos}

La gráfica Biplots GGE (Figura 3), muestra que los genotipos que más respondieron de manera positiva $o$ negativa a los estímulos ambientales por estar ubicados en las esquinas del polígono fueron: IDIAP 38, FLAR 198-00, FLAR 199-00, CHI 49-6, Prosequisa, FLAR 195-00, CHI 13-256, P-1048 y P-3621.

Cuadro 5. Análisis AMMI para rendimiento de grano de 20 cultivares de arroz evaluados en seis ambientes bajo condiciones de riego. IDIAP. Panamá. 2002.

\begin{tabular}{lrcrr}
\hline $\begin{array}{c}\text { Fuente de } \\
\text { variación }\end{array}$ & $\begin{array}{c}\text { Grados de } \\
\text { libertad }\end{array}$ & $\begin{array}{c}\text { Suma de } \\
\text { cuadrado }\end{array}$ & $\begin{array}{c}\text { Cuadrado \% explicado } \\
\text { medio }\end{array}$ & $\begin{array}{c}\text { por fuente } \\
\text { var. }\end{array}$ \\
\hline Modelo & 121 & 824,4218413 & $6,8134036^{* *}$ & \\
Ambientes (A) & 5 & 667,8514069 & 133,5702814 & 81,0 \\
Genotipos (G) & 19 & 49,0201047 & 2,5800055 & 6,0 \\
G x A & 95 & 96,6089290 & 1,0169361 & 12,0 \\
PCA1 & 23 & 37,9409 & 1,64960 & 43 \\
PCA2 & 21 & 19,2325 & 0,91583 & 20 \\
Error & 238 & 152,5550420 & 0,6409876 & \\
Total & 359 & 976,9768833 & & \\
\hline
\end{tabular}

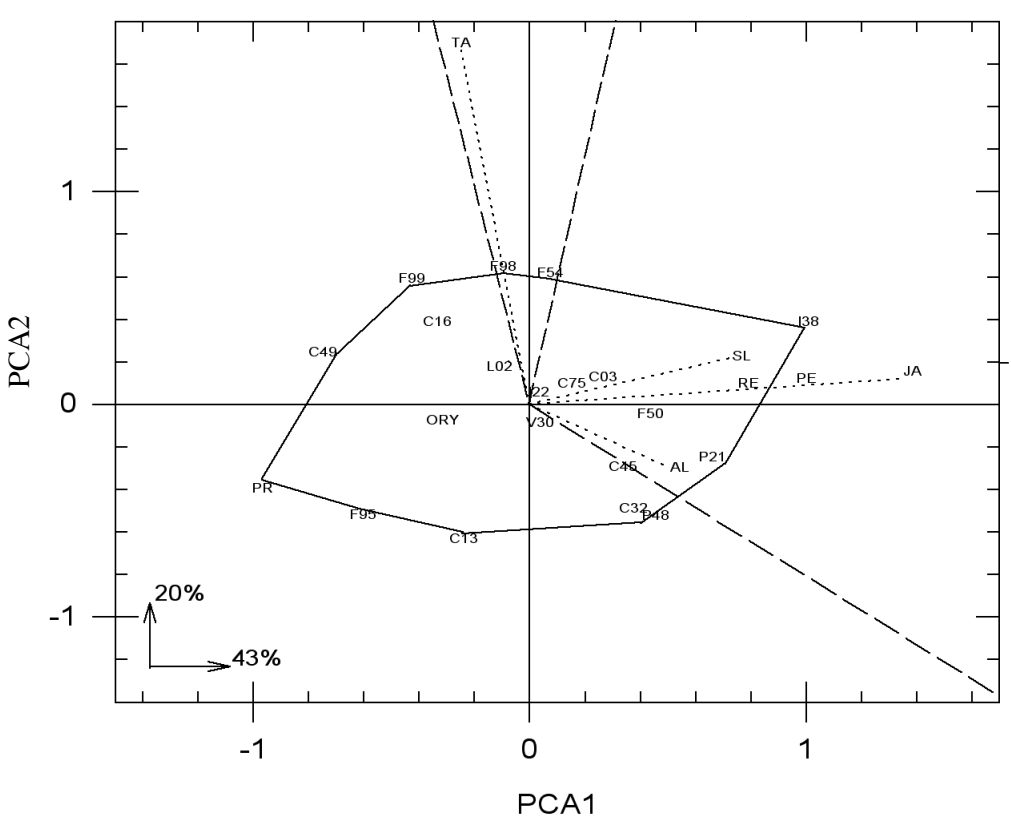

Figura 3. Puntuaciones del primer y segundo eje del componente principal de 20 cultivares de arroces intermedios en seis ambientes de Riego. IDIAP, Panamá, 2002. (GGE-Biplot). 
Los cultivares IDIAP 38, P-3621 y FLAR 54-00, fueron considerados por el modelo como los genotipos que mejor respondieron a los estímulos ambientales concepto de adaptabilidad de Lin et al. 1986, Lin y Binns 1994; estos materiales tuvieron buen desempeño en la mayoría de las localidades evaluadas.

Los cultivares Prosequisa, FLAR 195-00, CHI 496 y CHI 13-256, fueron los que presentaron el comportamiento más pobre en la mayoría de los ambientes, por su ubicación lejos de los sectores donde están las localidades. Los materiales con mayor estabilidad, bajo estas condiciones de riego fueron la variedad Fedearroz 50, IDIAP 38 y P-3621, por presentar puntuaciones altas en el PCA1 y puntuaciones cercanas a cero en el PCA2 (Figura 3).

\section{Efecto de los ambientes}

Los ambientes fueron agrupados en dos sectores, el primero conformado por cinco localidades: Alanje (AL), Jacú (JA), San Lorenzo (SL), Penonomé (PE) y Remedios (RE); este agrupamiento sugiere que tienen la misma capacidad discriminatoria, donde el genotipo superior fue IDIAP 38. En el otro sector se ubicó Tanara (TA), donde el FLAR 198-00 tuvo el mejor comportamiento.
Esta localidad mostró ser diferente a todas las demás localidades y se considera por la longitud de su vector como el mejor ambiente para evaluar puesto que discriminó los genotipos de manera diferente.

\section{Análisis de medias}

El análisis combinado de medias para algunas de las variables en estudio son presentadas en el Cuadro 6. Se observa que bajo el sistema de riego, la floración fluctuó entre 74ะ4 días en el FLAR 195-00 y 199-00 hasta $91 \pm 8$ días en Prosequisa; la media del ensayo estuvo en 81 días, o sea una precocidad de 13 días en relación con la media observada en el mismo juego de materiales bajo las condiciones secano.

Hubo baja incidencia de enfermedades como la piricularia al cuello de la panícula (NBl), del escaldado de la hoja (LSc) y Rhizoctonia (ShB. Los números entre los paréntesis indican la evaluación máxima alcanzada por estos genotipos al menos en un ensayo en una localidad específica.

Hubo diferencias significativas en rendimiento de grano, sobresaliendo las variedades IDIAP 38, P-3621 y Fedearroz 50, con rendimientos de 6.243, 5.949 y $5.796 \mathrm{t} / \mathrm{ha}$, respectivamente.

Cuadro 6. Rendimiento promedio t/ha (14\%) y otras características agronómicas de 20 cultivares de arroz de ciclo intermedio bajo el sistema de riego. Combinado de seis localidades. IDIAP. 2002.

\begin{tabular}{|c|c|c|c|c|c|c|c|c|c|c|}
\hline Cultivar & $\begin{array}{l}\text { HT } \\
(\mathrm{cm})\end{array}$ & $\begin{array}{c}\text { FL } \\
\text { (dds) }\end{array}$ & $\begin{array}{l}\text { NBL } \\
(1-9)\end{array}$ & $\begin{array}{l}\text { LSC } \\
(1-9)\end{array}$ & $\begin{array}{l}\text { SHB } \\
(1-9)\end{array}$ & $\begin{array}{c}\text { REND } \\
\text { (t/ha) }\end{array}$ & $\begin{array}{l}\text { RT } \\
(\%)\end{array}$ & $\begin{array}{l}\text { GE } \\
(\%)\end{array}$ & $\begin{array}{c}\text { CB } \\
(1-5)\end{array}$ & $\begin{array}{c}\text { DA } \\
(1-7)\end{array}$ \\
\hline IDIAP 38 & $95 \pm 9$ & $81 \pm 4$ & $2(3)$ & $2(4)$ & $2(5)$ & $6.243 \pm 1.93$ & $68,7 \pm 3,5$ & $57,5 \pm 6,9$ & $1,7 \pm 0,2$ & $5,8 \pm 1,7$ \\
\hline P-3621 & $99 \pm 9$ & $89 \pm 8$ & $2(4)$ & $1(3)$ & $2(5)$ & $5.949 \pm 1,80$ & $67,9 \pm 1,8$ & $58,3 \pm 5,5$ & $1,6 \pm 0,2$ & $5,6 \pm 1,9$ \\
\hline FEDEARROZ 50 & $101 \pm 9$ & $79 \pm 4$ & $2(3)$ & $2(5)$ & $2(3)$ & $5.796 \pm 1,64$ & $67,8 \pm 5,0$ & $60,6 \pm 8,3$ & $2,0 \pm 0,7$ & $6,9 \pm 0,1$ \\
\hline FLAR 54-00 & $100 \pm 10$ & $77 \pm 3$ & $1(3)$ & $2(3)$ & $2(5)$ & $5.732 \pm 1,36$ & $70,2 \pm 2,3$ & $61,1 \pm 4,9$ & $2,0 \pm 0,5$ & $7,0 \pm 0,0$ \\
\hline FLAR 198-00 & $100 \pm 11$ & $76 \pm 3$ & $2(3)$ & $2(3)$ & $2(5)$ & $5.594 \pm 1,46$ & $70,7 \pm 2,8$ & $62,0 \pm 5,1$ & $2,1 \pm 0,5$ & $7,0 \pm 0,0$ \\
\hline CHI 45-23 & $96 \pm 8$ & $83 \pm 5$ & $2(3)$ & $2(6)$ & $2(5)$ & $5.584 \pm 1,76$ & $66,1 \pm 3,9$ & $51,5 \pm 11,3$ & $1,6 \pm 0,3$ & $4,2 \pm 0,2$ \\
\hline CHI 3-30 & $105 \pm 8$ & $86 \pm 6$ & $2(3)$ & $2(5)$ & $2(5)$ & $5.577 \pm 1,87$ & $67,3 \pm 2,7$ & $58,1 \pm 5,9$ & $1,7 \pm 0,4$ & $4,1 \pm 0,3$ \\
\hline CHI 32-10 & $102 \pm 11$ & $84 \pm 4$ & $2(3)$ & $2(5)$ & $2(5)$ & $5.538 \pm 2,01$ & $68,5 \pm 3,9$ & $55,2 \pm 8,9$ & $2,6 \pm 0,4$ & $4,5 \pm 1,4$ \\
\hline CHI 75-25 & $95 \pm 9$ & $79 \pm 4$ & $2(4)$ & $2(5)$ & $2(5)$ & $5.518 \pm 1,63$ & $67,1 \pm 3,3$ & $54,2 \pm 8,1$ & $2,5 \pm 0,7$ & $6,7 \pm 0,5$ \\
\hline LAJAS 02-00 & $102 \pm 7$ & $77 \pm 2$ & $2(4)$ & $2(5)$ & $2(5)$ & $5.470 \pm 1,30$ & $68,0 \pm 2,7$ & $59,5 \pm 5,3$ & $1,4 \pm 0,2$ & $5,0 \pm 0,4$ \\
\hline P-1048 & $101 \pm 10$ & $82 \pm 4$ & $2(3)$ & $2(5)$ & $2(5)$ & $5.454 \pm 2,14$ & $68,7 \pm 2,4$ & $57,3 \pm 5,4$ & $2,5 \pm 0,8$ & $7,0 \pm 0,0$ \\
\hline IDIAP 22 & $103 \pm 6$ & $88 \pm 7$ & $1(2)$ & $1(2)$ & $1(3)$ & $5.408 \pm 1,84$ & $69,7 \pm 5,2$ & $61,5 \pm 10,9$ & $2,8 \pm 0,3$ & $7,0 \pm 0,0$ \\
\hline VIORH 30 & $105 \pm 11$ & $83 \pm 4$ & $1(3)$ & $2(4)$ & $2(3)$ & $5.335 \pm 1,69$ & $67,0 \pm 4,3$ & $56,2 \pm 6,5$ & $2,0 \pm 0,6$ & $4,7 \pm 1,2$ \\
\hline CHI 16-12 & $102 \pm 10$ & $79 \pm 4$ & $2(4)$ & $2(5)$ & $2(3)$ & $5.319 \pm 1,41$ & $67,5 \pm 3,7$ & $56,4 \pm 5,3$ & $2,8 \pm 0,8$ & $5,7 \pm 1,8$ \\
\hline FLAR 199-00 & $98 \pm 9$ & $74 \pm 3$ & $2(4)$ & $2(4)$ & $2(4)$ & $5.253 \pm 1,58$ & $68,9 \pm 4,8$ & $58,9 \pm 7,8$ & $1,8 \pm 0,3$ & $7,0 \pm 0,0$ \\
\hline ORYZICA 1 & $96 \pm 10$ & $75 \pm 4$ & $2(3)$ & $2(4)$ & $2(5)$ & $5.108 \pm 1,69$ & $70,9 \pm 2,1$ & $63,6 \pm 4,8$ & $1,3 \pm 0,2$ & $6,2 \pm 1,2$ \\
\hline CHI 13-256 & $103 \pm 10$ & $80 \pm 4$ & 2(4) & $2(5)$ & $2(5)$ & $5.054 \pm 1,78$ & $65,8 \pm 2,3$ & $54,0 \pm 4,8$ & $1,9 \pm 0,6$ & $4,8 \pm 0,1$ \\
\hline CHI 49-6 & $106 \pm 9$ & $87 \pm 7$ & $1(2)$ & $2(4)$ & $2(5)$ & $5.002 \pm 1,14$ & $67,2 \pm 5,2$ & $59,1 \pm 8,4$ & $1,5 \pm 0,2$ & $4,5 \pm 0,0$ \\
\hline FLAR 195-00 & $101 \pm 9$ & $74 \pm 4$ & $1(3)$ & $2(4)$ & $2(5)$ & $4.943 \pm 1,21$ & $69,9 \pm 2,0$ & $61,0 \pm 3,0$ & $1,2 \pm 0,3$ & $6,9 \pm 0,1$ \\
\hline PROSEQUISA & $122 \pm 10$ & $91 \pm 8$ & $1(2)$ & $2(5)$ & $2(5)$ & $4.560 \pm 1,36$ & $66,7 \pm 5,5$ & $56,2 \pm 10,1$ & $1,6 \pm 0,4$ & $3,9 \pm 0,1$ \\
\hline Promedio & 102 & 81 & 2 & 2 & 2 & 5.422 & 68,2 & 58,1 & 1,9 & 5,7 \\
\hline DMS & 3 & 2 & 0,3 & 0,3 & 0,6 & 0,485 & & & & \\
\hline
\end{tabular}


Bajo las condiciones de riego los genotipos presentaron mejores parámetros de calidad de grano, con porcentajes de rendimiento total (RT) varió entre 65,8 a $70,9 \%$, con valores de granos enteros (GE) entre 51,5 y $62,0 \%$. La incidencia de centro blanco (CB) en los granos fluctuó entre 1,2 y 2,8 en la escala de uno a cinco, en total seis cultivares presentaron calificación de centro blanco superior a dos, estos valores de CB están relativamente altos de acuerdo a los parámetros de selección del proyecto de investigación de arroz. Cabe resaltar a las líneas avanzadas FLAR 54-00 y FLAR 198-00 por su rendimiento y calidad de grano.

En cuanto a la digestión alcalina (DA), el $45 \%$ de los genotipos presentaron grados de dispersión de seis y siete, un $50 \%$ con grado de cuatro y cinco y $5 \%$ con grado inferior a cuatro (Arregocés y Zamorano 1979).

La interpretación de la interacción genotipo por ambiente a través del modelo AMMI, permitió verificar que bajo condiciones de secano la magnitud de la suma de cuadrados de la interacción $\mathrm{G}$ x A, fue 4,7 veces mayor que la de genotipo, mientras que bajo condiciones de riego fue apenas dos veces mayor, indicando que los ambientes de secano tuvieron más influencia en las respuestas diferenciales de los genotipos que los ambientes de riego, lo cual es una respuesta obvia por la naturaleza de la planta de arroz.

Por otro lado, cuando se compara el comportamiento de los genotipos en los dos sistemas de manejo, secano y riego, fue evidente la respuesta diferencial de algunos cultivares. En este sentido, se registraron diferencias en la respuestas de los cultivares ante las enfermedades, rendimiento de grano y obviamente en las variables de calidad de grano. Sin embargo, los mismos tres genotipos fueron identificados como superiores en ambos sistemas, por combinar alto potencial de rendimiento, con buen rendimiento industrial de granos enteros: FLAR 54-00, IDIAP 38 y LAJAS02-00. Debido a la adaptabilidad mostrada por estos genotipos en los diferentes ambientes y sistemas, se sugiere continuar su evaluación para verificar este buen comportamiento.

Es importante recalcar que en secano el Biplot $\mathrm{GGE}_{\mathrm{SREG}}$, determinó que las líneas con mejor estabilidad fueron FLAR 198-00, Fedearroz 50 y FLAR 54-00; esta última fue la que mejor respondió a los estímulos ambientales (adaptabilidad). Los ambientes más discriminatorios de los genotipos fueron Penonomé, Barú y Calabacito. No obstante, en riego los cultivares con mejor estabilidad fueron Fedearroz 50, IDIAP 38 y P3621. Por otro lado, Tanara fue la localidad que mejor discriminó los genotipos evaluados. La ventaja del modelo Biplot $\mathrm{GGE}_{\mathrm{SREG}}$, de identificar los ambientes que mejor discriminan los genotipos, posiblemente en el tiempo permita ser más eficiente en el trabajo de evaluación de cultivares, porque permitiría priorizar las evaluaciones en estas localidades.

\section{LITERATURA CITADA}

ARREGOCÉS, O.; A. DE ZAMORANO. 1979. Evaluación de la calidad del arroz. Audiotutorial CIAT, 24 p.

CROSSA, J. 1990. Statistical analysis of multi location trials. Advances in agronomy 44: 55-85.

CROSSA, J.; GAUCH, H.G. JR.; ZOBEL, R.W. 1990. Additive main effects and multiplicative interaction analysis of two international maize cultivar trials. Crop Sci. 30:493-500.

CROSSA, J.; FOX, P.N.; PFEIFFER, W.H.; RAJARAM, S.; GAUCH, H.G. Jr. 1991. AMMI adjustment for statistical analysis of an international wheat yield trial. Theor. Appl. Genet. 81:27-37.

GAUCH, H. G.; ZOBEL, R.W. 1988. Predictive and postdictive success of statistical analysis of yield trials. Theor. Appl. Genet. 76:1-10

KAYA, Y.; PALTA, C.; TANER, S. 2002. Additive main effects and multiplicative interactions analysis of yield performances in bread wheat genotypes across environments. Turk J. Agric for 26:275-279.

LIN C.S.; BINNS, M.R. 1994. Concepts and methods for analyzing regional trial data for cultivar and location selection. Plant Breeding Reviews 12:271-297.

LIN, C.S.; BINNS, M.R.; LEFKOVITH, L.P. 1986. Stability analysis. Where do we stand? Crop Sci. 26.894-900.

MIDA (Ministerio de Desarrollo Agropecuario). 2003. Dirección Nacional de Agricultura. Informe final sobre la superficie sembrada de arroz y producción nacional para el año agrícola 2002-2003.

YAN, W, L.; HUNT, A.; SHENG, Q.; SZLAVNICS, Z. 2000. Cultivar evaluation and mega environment investigation based on the GGE Biplot. Crop Sci. 40:597-605.

YAN, W.; CORNELIUS, P.; CROSSA, J.; HUNT, L.A. 2001. Two types of GGE Biplots for analyzing multi - environment trial data. Crop Sci. 41:656-663.

YAN, W, L.; HUNT, A. 2002. Biplots analysis of multi - environment trial data. $\mathrm{CAB}$ International. Quantitative Genetics, Genomics and Plant Breeding. Ed. M.S. Kang. p. 289-303.

YAN, W.; RAJCAN, I. 2002. Biplot analysis of test sites and trait relations of soybean in Ontario. Crop Sci. 42:11-20.

ZOBEL R.W.; WRIGHT, M.J.; GAUCH, H.G. Jr. 1988. Statistical analysis of a yield trial. Agron. J. 80:388-393.

AGRONOMÍA MESOAMERICANA 16(2): 117-125. 2005 